\title{
Coexpression of Arp2 and WAVE2 predicts poor outcome in invasive breast carcinoma
}

\author{
Keiichi Iwaya ${ }^{1}$, Kohno Norio ${ }^{2}$ and Kiyoshi Mukai ${ }^{1}$ \\ ${ }^{1}$ Department of Diagnostic Pathology, Tokyo Medical University, Shinjuku-ku, Tokyo, Japan and \\ ${ }^{2}$ Department of Breast Cancer, Tokyo Medical University, Shinjuku-ku, Tokyo, Japan
}

\begin{abstract}
Breast carcinoma with a high histologic grade is highly invasive and metastatic. One reason for its irregular morphology is the formation of excessive protrusions due to assemblages of branched actin filament networks. In mammalian cells, the actin-related protein 2 and 3 (Arp2/3) complex initiates actin assembly to form lamellipodial protrusions by binding to the Wiskott-Aldrich syndrome (WASP)/WASP family verprolinehomologous protein2 (WAVE2), a member of the WASP protein family. In this study, the localization Arp2 and WAVE2 in breast carcinoma was investigated to clarify whether coexpression of the two proteins is associated with histologic grade or patient outcome. Immunohistochemical staining of Arp2 and WAVE2 was performed on mirror specimens of 197 breast carcinomas, and the association between coexpression of the two proteins and clinicopathologic factors was examined. Kaplan-Meier disease-free survival and overall survival curves were analyzed to determine the prognostic significance of Arp2 and WAVE2 coexpression in breast carcinoma. Coexpression of Arp2 and WAVE2 was detected in 64 (36\%) of 179 invasive ductal carcinomas and in 2 (11\%) of 18 ductal carcinomas in situ, but was not detected in any adjacent non-cancerous tissue. The proportion of cancer cells expressing both Arp2 and WAVE2 was significantly higher in cases with high histologic grade $(P<0.0001)$, and cases with lymph node metastasis $(P=0.0150)$. The patients whose cancer cells showed such coexpression had shorter disease-free $(P=0.0002)$ and overall survival $(P=0.0122)$ than patients whose cancer cells expressed only one or none of Arp2 and WAVE2. Multivariate Cox regression analysis revealed that coexpression of Arp2 and WAVE2 is an independent factor for both tumor recurrence $(P=0.0308)$ and death $(P=0.0455)$. These results indicate that coexpression of Arp2 and WAVE2 is a significant prognostic factor that is closely associated with aggressive morphology of invasive ductal carcinoma of the breast.

Modern Pathology (2007) 20, 339-343. doi:10.1038/modpathol.3800741; published online 2 February 2007
\end{abstract}

Keywords: invasion; actin; polymerization; breast cancer; histologic grade

Actin is one of the most abundant proteins in eukaryotes, being a component of the cytoskeleton that determines and maintains cell shape. Eukaryotic cells can change their shape dramatically and migrate as a result of actin polymerization and/or depolymerization. Cell migration is initiated by plasma membrane protrusions, in the form of filopodia and lamellipodia, which are also known as invadopodia in the case of cancer cells. Among the variously shaped membrane protrusions, flattened lamellipodia are likely to play the most important role in directional movement. ${ }^{1}$

Lamellipodia are composed of dense networks of actin filaments that are branched at about $70^{\circ}$. The

Correspondence: Dr K Iwaya, MD, Department of Diagnostic Pathology, Tokyo Medical University, Nishi-Shinjuku 6-7-1, Shinjuku-ku, Tokyo 160-0023, Japan.

E-mail: kiwaya@tokyo-med.ac.jp

Received 10 October 2006; revised and accepted 27 November 2006; published online 2 February 2007 actin-related protein 2 and 3 complex (Arp2/3 complex) is present at the corners of branched actin filaments, and is indispensable for lamellipodium formation. ${ }^{2,3}$ The Arp2/3 complex rapidly catalyzes actin filament nucleation and elongation, and is also essential for efficient remodeling of actin filaments by ATP hydrolysis. ${ }^{4-6}$

The Arp2/3 complex is activated by binding to the Wiskott-Aldrich syndrome protein (WASP) family proteins. There are five WASP family proteins; WASP, neural WASP (N-WASP), and WASP family verprolin-homologous protein (WAVE)1-3, known to be a suppressor of cAMP-receptors (SCAR1-3). They are very potent cellular nucleating factors, and share a common C-terminal verprolin-homologyconnecting acidic (VCA) domain that is responsible for Arp2/3 complex activation. However, WASP and WAVE proteins differ in both the signaling inputs they receive and their cellular outputs, which include adhesion, endocytosis, trafficking, and migration. 
Among the WASP family proteins, WAVE2 has a central role in the formation of lamellipodia and the initiation of amoeboid movement. ${ }^{7,8}$ Interaction of the Arp2/3 complex and WAVE2 occurs at the leading edge downstream in the signaling pathway responsible for directional movements in response to various stimuli such as epidermal growth factor (EGF) and platelet-derived growth factor (PDGF). ${ }^{7,9}$ Activation of these receptors generates phosphatidylinositol $^{3-5}$ triphosphate (PIP3), which in turn activates Rac on the cell membrane. WAVE2 is a PIP3-binding protein and can regulate activation of the Arp2/3 complex from the signals of PIP3 and activated Rac. ${ }^{10}$

The majority of WAVE2 in the cell is complexed with Abl-interactor-1 (Abi1), Nck-associated protein-1 (Nap1), a small protein HSPC300, and p53inducible protein-121 (PIR121)/Sra1. ${ }^{11}$ Although the activity of the WAVE2 complex has yet to be examined, it is considered to be strictly regulated by other molecules that act as different signaling inputs regulating actin dynamics.

Thus, the binding of the Arp2/3 complex and WAVE2 is a final intracellular signal that initiates actin polymerization for the formation of lamellipodial protrusions. The signal is considered to be integrated by various signaling inputs. In order to clarify the clinical significance of this cellular signal, we previously examined the coexpression of Arp2 and WAVE2 in human lung adenocarcinoma by immunostaining using mirror specimens, and the coexpression was found to be correlated with poorer patient outcome. ${ }^{12}$ In the present study, immunostaining of Arp2 and WAVE2 was performed in specimens of breast cancer and the correlation of coexpression of these proteins with histologic grade and patient outcome was examined.

\section{Materials and methods}

\section{Study Subjects}

The subjects of this study were 197 Japanese women who underwent modified radical mastectomy for breast cancer at the Tokyo Medical University Hospital between 1988 and 2002. The median follow-up period for all patients was 7.7 years, and that of living patients was 8.6 years (range, 3.5-13.6 years). As adjuvant chemotherapy between 1988 and 1989, 63 patients of invasive ductal carcinoma received orally a combination of uracil and tegafur, often referred to as UFT, (250 mg of tegafur per square meter of body-surface area per day) for two years after the surgery. From 1990 to 2002 UFT plus tamoxifen $(20 \mathrm{mg} /$ day $)$ were given to the patients with ER-positive invasive ductal carcinoma and only oral UFT for those with ER-negative invasive ductal carcinoma. Other 18 patients of ductal carcinoma in situ did not receive any chemotherapy. Blocks of formalin-fixed, paraffin-embedded tissue from these patients were retrieved from the Depart- ment of Diagnostic Pathology, and clinicopathological data, such as age at the time of diagnosis, tumor size, and axillary lymph node status, were obtained from the medical records. To confirm the presence of sufficient tumor tissue and to ensure contemporary uniform grading, paraffin-embedded tissue blocks were sectioned, stained with hematoxylin-eosin, reviewed for the presence of tumor, and histological grade was determined by a single pathologist, as outlined previously. ${ }^{13}$ Informed consent was obtained from each patient.

\section{Immunohistochemistry}

One or two representative blocks of each tumor were selected for immunohistochemical analysis of Arp2 and WAVE2. From all paraffin blocks, 4- $\mu \mathrm{m}$-thick sections were cut and placed on silane-coated slides. In order to clarify the localization of Arp2 and WAVE2, we prepared mirror sections from a pair of consecutive specimens. These were placed on slides with the common cross-section turned upwards, so that they would share the same cutting surface. The features in such specimens appear reversed, and the specimens are therefore known as mirror specimens. Immunohistochemical procedure was described in our previous paper. ${ }^{12}$ Briefly, antigen retrieval was achieved by heating the slides in an autoclave at $110^{\circ} \mathrm{C}$ for $10 \mathrm{~min}$ in $1 \mathrm{mM}$-EDTA (pH8.0). After cooling, pairs of mirror specimens were incubated overnight at room temperature with the primary antibody, goat polyclonal anti-human Arp2 antibody, at a dilution of 1:50 (k-15, Santa Cruz Biotechnology, Santa Cruz, CA, USA) and goat polyclonal anti-human WAVE2 antibody at a dilution of 1:50 (c-14, Santa Cruz Biotechnology), respectively.

If more than $5 \%$ of cancer cells expressed both Arp2 and WAVE2 in their cytoplasm, Arp2 and WAVE2 were judged to be positive for coexpression in the tumor.

\section{Statistical Analysis}

Correlations between the coexpression of Arp2 and WAVE2 and clinicopathologic factors were evaluated by $\chi^{2}$ tests, at a significance level of $P<0.05$. Univariate and multivariate survival analyses were performed to evaluate the prognostic significance of coexpression and the effect of other factors, such as age, tumor size, axillary lymph node status, and histologic grade, on the clinical outcome.

\section{Results}

\section{Immunohistochemistry}

Coexpression of Arp2 and WAVE2 was detected in $64(36 \%)$ of 179 invasive ductal carcinomas and in 2 (11\%) of 18 ductal carcinomas in situ, and there was 
Table 1 Association between the coexpression of Arp2 and WAVE2 and pathologic factors

\begin{tabular}{lccc}
\hline Factors & Cases & Coexpression (\%) & P-value \\
\hline Tumor size & & & \\
T1 & 59 & $16(27)$ & \\
T2 & 99 & $71(41)$ & NS \\
T3 & 21 & & \\
& & & \\
State of axillary lymph node & 68 & $29(28)$ & 0.015 \\
$\quad$ Metastasis & 102 & & \\
No metastasis & & $1(4)$ & \\
& & $27(29)$ & \\
Histologic grade & 25 & $36(59)$ & \\
$\quad$ Grade 1 & 93 & & \\
Grade 2 & 61 & $64(11)$ & 0.0001 \\
Grade 3 & & & \\
Invasive type & 18 & & \\
DCIS & 179 & & \\
IDC & & & \\
\end{tabular}

a significant difference in the frequency of coexpression between invasive carcinoma and noninvasive carcinoma (Table $1, P=0.0333$ ). The cancer cells showed coexpression most frequently at the invading front (Figure 1). In most cases judged positive for the coexpression, number of cancer cells expressing both Arp2 and WAVE2 were no more that $10 \%$ of whole cancer cells. Either Arp2 or WAVE2 was focally stained in normal epithelial cells or stromal cells, and no normal cells showed distinct coexpression of Arp2 and WAVE2 except for macrophages.

\section{Association between Coexpression and Clinicopathologic Factors}

Among 61 invasive ductal carcinomas with a high histologic grade (grade 3), 36 (59\%) were coexpression-positive (Table 1). However, 27 (29\%) of 93 grade 2 and 1 ( $4 \%$ ) of 25 grade 1 tumors were judged to be positive for localization. Thus the coexpression was strongly associated with a high histologic grade $(P<0.0001)$.

Cancer cells with positive coexpression frequently metastasized to the axillary lymph nodes $(P=0.0150)$. There was no correlation between coexpression and $\mathrm{T}$ factor.

Table 2 shows the association between coexpression and established biologic markers of breast cancer. Coexpression was strongly associated with positive expression of Her2/neu $(P<0.0001)$. It was inversely correlated with both estrogen $(P=0.0027)$ and progesterone receptors $(P=0.0025)$. Nuclear p53 immunoreactivity was not correlated with coexpression in this study.

\section{Association between Coexpression and Patient Outcome}

Figure 2 shows the Kaplan-Meier disease-free survival (DFS) curves of patients with tumors

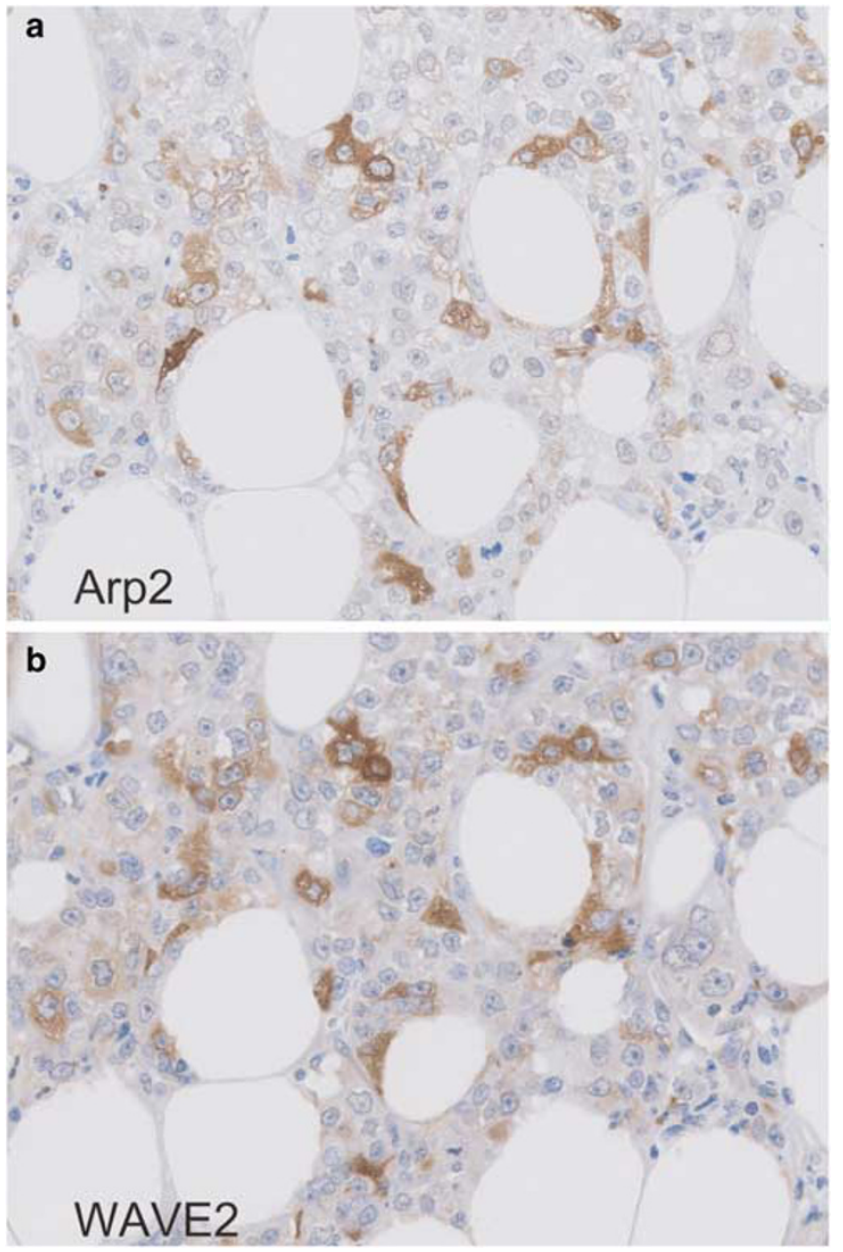

Figure 1 A case of invasive ductal carcinoma in which mirror sections were stained for Arp2 and WAVE2. Specimens A was stained for Arp2, and the corresponding mirror sections B was stained for WAVE2. Staining of both Arp2 and WAVE2 are present homogeneously in the cytoplasm of the same cancer cells.

showing positive coexpression and those negative for coexpression. Their DFS curves showed a significant difference as estimated by the log-rank test $(P=0.0002)$. Similarly, histologic grade and axillary lymph node status were significant prognostic factors for DFS. Among them, multivariate Cox regression analysis demonstrated that coexpression of Arp2 and WAVE2 $(P=0.0002)$ and axillary lymph node status had independent prognostic value for DFS $(P<0.0155)$ (Table 3$)$.

There was also a significant difference in the overall survival curves between the positive and negative groups (Figure $2, P=0.0122$ ), and coexpression was found to be an independent prognostic factor for overall survival by multivariate analysis (Table 4, $P=0.0455$ ). Of 63 invasive ductal carcinoma cases between 1988 and 1989 that were given oral UFT as adjuvant chemotherapy, cases with positive coexpression had shorter disease-free $(P<0.0001)$ and overall survival $(P=0.0002)$ than those without coexpression of Arp2 and WAVE2. 
Table 2 Association between coexpression of Arp2 and WAVE2 and biologic factors

\begin{tabular}{lrcc}
\hline Factors & Cases & Coexpression (\%) & P-value \\
\hline $\begin{array}{l}\text { Her2/neu } \\
\text { 0 and 1+ }\end{array}$ & 132 & $32(24)$ & \\
2+ and 3+ & 47 & $32(68)$ & $<0.0001$ \\
ER & & & \\
$\quad$ Negative & 58 & $30(52)$ & 0.0027 \\
Positive & 121 & $34(28)$ & \\
PR & & & 0.0025 \\
Negative & 73 & $36(49)$ & \\
Positive & 106 & $28(26)$ & 0.1408 \\
$p 53$ & & & \\
$\quad$ Negative & 119 & $38(32)$ & \\
Positive & 60 & $26(43)$ & \\
\hline
\end{tabular}
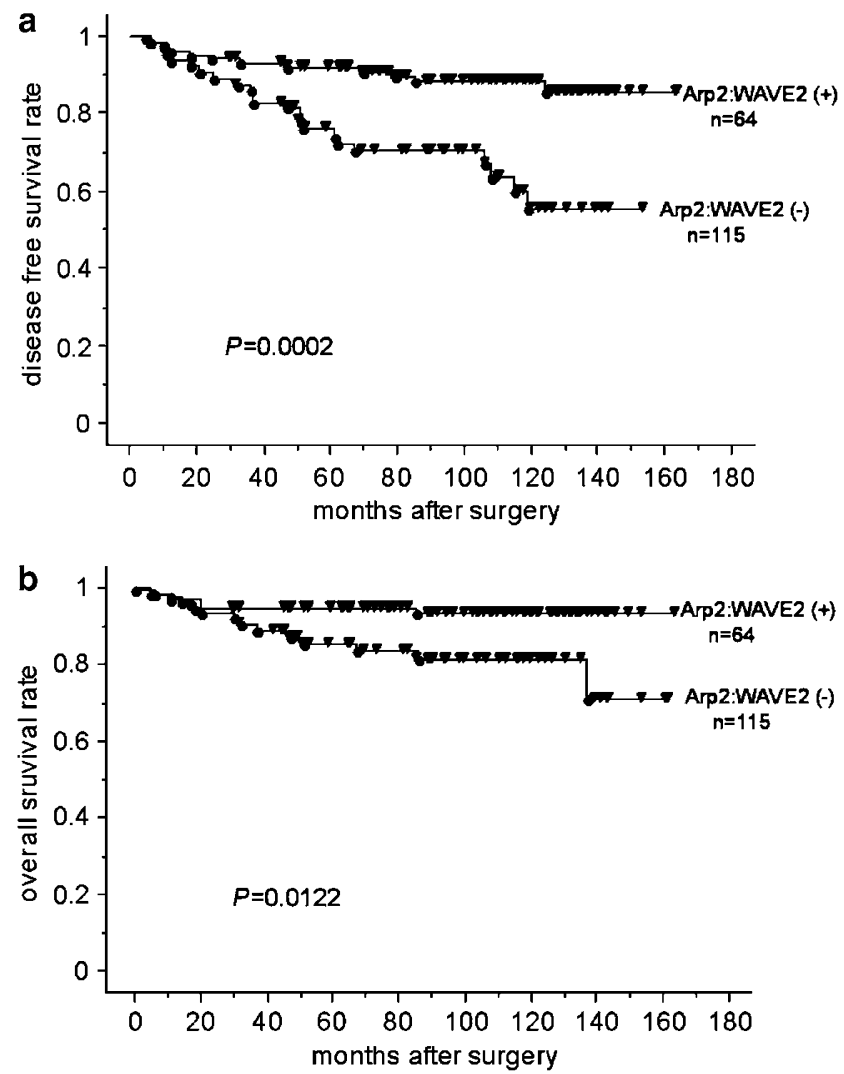

Figure 2 Kaplan-Meier analysis in the Arp2 and WAVE2 coexpression-positive group and -negative group. (a) KaplanMeier DFS curves for 179 patients stratified by an Arp2 and WAVE2 coexpression-positive group and -negative group. (b) Kaplan-Meier overall survival curves for 179 patients in an Arp2 and WAVE2 coexpression-positive group and -negative group. $P$-values were calculated using the log-rank test.

Similarly, 116 patients between 1990 and 2002 with coexpression had shorter disease-free $(P=0.0317)$, however, there was no difference in overall survival $(P=0.1832)$ between two groups.

\section{Discussion}

The physiological role of WAVE2 bound to the Arp2/3 complex in the formation of lamellipodia, which initiate migration, has been established. ${ }^{14}$ In the present study, we demonstrated coexpression of Arp2 and WAVE2 in breast cancer tissue. As a preliminary experiment, we prepared cell blocks of breast cancer cell lines such as MCF7, SK-BR-3, and AU-565, and subjected them to Arp2 and WAVE2 immunostaining. The staining pattern of Arp2 and WAVE2 coexpression in these cell lines was predominant at the leading edge, as revealed by double staining and confocal laser scanning microscopy. We considered that such coexpression in breast cancer cells corresponds to binding of WAVE2 to the Arp2/3 complex at the leading edge, and that this is at least partly involved in cancer cell migration that facilitates invasion and metastasis. Our findings that the coexpression was frequently detected at the invading front and was significantly correlated with axillary lymph-node metastasis support the involvement of coexpression in the process of invasion and/ or metastasis.

This study revealed that WAVE2:Arp2/3 coexpression is an independent prognostic factor of both DFS and overall survival, and functionally represents a signal of lamellipodium formation and cell migration. Such coexpression was detected in 59\% of carcinomas with high histologic grade. Excessive formation of lamellipodial protrusions is suggested to induce an aggressive morphology and active movement of cancer cells. However, ductal carcinomas with high histologic grade also contain cancer cells with a round shape. Other signals of cellular migration such as the Rho/ROCK pathway and expression of cortactin may be enhanced in such cancer cells. ${ }^{15-18}$ If it were possible to eliminate both signals of cellular migration, then the ability of cancer for invasion might become easier to understand.

WAVE2:Arp2/3 coexpression is considered to be an intracellular signal occurring downstream of tyrosine kinase receptors such as EGF and PDGF. Our data showed that such coexpression is significantly associated with Her2/neu overexpression, suggesting that the coexpression is also downstream from Her2/neu stimuli. Currently, trastuzumab is a powerful therapeutic agent for advanced breast cancer. WAVE2:Arp2/3 coexpression may eliminate the ability of trastuzumab to suppress invasion and/or metastasis, although further examination to confirm the direct link between Her2/neu overexpression and coexpression is necessary.

The coexpression of Arp2 and WAVE2 was detected most frequently at the invading front, and was not present diffusely throughout the cancer tissue. In human breast cancer cell lines, EGF stimulation does not induce WAVE2:Arp2/3 coexpression in all cells but only in a proportion of them. These findings suggest that WAVE2:Arp2/3 
Table 3 Multivariate conditional logistic regression analysis for prediction of tumor recurrence

\begin{tabular}{lccc}
\hline \multirow{2}{*}{ Factor } & $\begin{array}{c}\text { Univariate } \\
\text { P-value }\end{array}$ & \multicolumn{2}{c}{ Multivariate } \\
\cline { 3 - 4 } & & $\begin{array}{c}\text { Hazard } \\
\text { rate }\end{array}$ & P-value \\
& & & \\
\hline Axillary lymph node status & 0.0155 & 3.939 & 0.0472 \\
$\begin{array}{l}\text { Histologic grade, G1 and G2 vs G3 } \\
\text { Arp2-WAVE2, negative vs positive }\end{array}$ & 0.0089 & & \\
& & &
\end{tabular}

Table 4 Multivariate conditional logistic regression analysis for prediction of tumor death

\begin{tabular}{lcccc}
\hline \multirow{2}{*}{ Factor } & $\begin{array}{c}\text { Univariate } \\
\text { P-value }\end{array}$ & \multicolumn{2}{c}{ Multivariate } \\
\cline { 3 - 5 } & & \multicolumn{2}{c}{$\begin{array}{c}\text { Hazard } \\
\text { rate }\end{array}$} & P-value \\
& & & \\
\hline ER, negative vs positive & 0.0469 & & \\
Arp2-WAVE2, negative vs positive & 0.0122 & 2.66 & 0.0455
\end{tabular}

coexpression is regulated by other stimuli, for example, cell-to-cell contact, and that cancer cells appear to be enhanced by coexpression because adjacent non-cancerous breast epithelial cells showed no coexpression. Clarification of the function of the WAVE2 complex may be a key to resolving this issue and for finding molecules that could be useful as therapeutic targets.

We propose that coexpression of Arp2 and WAVE2 is a new prognostic factor in breast cancer, which partly reflects cell migration ability. Such coexpression may prove promising for clinical manipulation with a view to future therapy.

\section{Acknowledgements}

This study was supported in part by a Grant-in-Aid for Scientific Research (C) (Kakenhi 15590319). We thank Dr Tadaomi Takenawa of Department of Biochemistry, Institute of Medical Science, University of Tokyo for helpful suggestions and discussion.

\section{References}

1 Lauffenburger DA, Horwitz AF. Cell migration: a physically integrated molecular process. Cell 1996;84: 359-369.

2 Amann KJ, Pollard TD. Direct real-time observation of actin filament branching mediated by Arp $2 / 3$ complex using total internal reflection fluorescence microscopy. Proc Natl Acad Sci USA 2001;98:15009-15013.
3 Fujiwara I, Suetsugu S, Uemura S, et al. Visualization and force measurement of branching by Arp2/3 complex and N-WASP in actin filament. Biochem Biophysics Res Commun 2002;293:1550-1555.

4 Welch MD, Iwamatsu A, Mitchison TJ. Actin polymerization is induced by Arp2/3 protein complex at the surface of Listeria monocytogenes. Nature 1997; 385:265-269.

5 Schafer DA, Welch MD, Machesky LM, et al. Visualization and molecular analysis of actin assembly in living cells. J Cell Biol 1998;143:1919-1930.

$6 \mathrm{Lu}$ Clainche C, Pantaloni D, Carlier MF. ATP hydrolysis on actin-related protein $2 / 3$ complex causes debranching of dendritic actin arrays. Proc Natl Acad Sci USA 2003;100:6337-6342.

7 Suetsugu S, Yamazaki D, Kurisu S, et al. Differential roles of WAVE1 and WAVE2 in dorsal and peripheral ruffle formation for fibroblast cell migration. Dev Cell 2003;5:595-609.

8 Yamazaki D, Suetsugu S, Miki $\mathrm{H}$, et al. WAVE2 is required for directed cell migration and cardiovascular development. Nature 2003;424:452-456.

9 Bailly M, Macaluso F, Cammer M, et al. Relationship between Arp2/3 complex and the barbed ends of actin filaments at the leading edge of carcinoma cells after epidermal growth factor stimulation. J Cell Biol 1999; 145:331-345.

10 Oikawa T, Yamaguchi $\mathrm{H}$, Itoh $\mathrm{T}$, et al. PtdIns $(3,4,5) \mathrm{P} 3$ binding is necessary for WAVE2-induced formation of lamellipodia. Nat Cell Biol 2004;6:420-426.

11 Kunda P, Craig G, Dominguez V, et al. Abi1, Sra1, and Kette control the stability and localization of SCAR/ WAVE to regulate the formation of actin-based protrusions. Curr Biol 2003;13:1867-1875.

12 Semba S, Iwaya K, Matsubayashi J, et al. Coexpression of actin-related protein 2 and Wiskott-Aldrich syndrome family verproline-homologous protein 2 in adenocarcinoma of the lung. Clin Cancer Res. 2006;12: 2449-2454.

13 Elston CW, Ellis IO. Pathological prognostic factors in breast cancer. I. The value of histological grade in breast cancer: experience from a large study with longterm follow-up. Histopathology 1991;19:403-410.

14 Yamazaki D, Fujiwara T, Suetsugu S, et al. A novel function of WAVE in lamellipodia: WAVE1 is required for stabilization of lamellipodial protrusions during cell spreading. Genes Cells 2005;10:381-392.

15 Sahai E, Marshall CJ. Differing models of tumor cell invasion have distinct requirements for Rho/ROCK signaling and extracellular proteolysis. Nat Cell Biol 2003;5:711-719.

16 Hotulainen P, Lappalainen P. Stress fibers are generated by two distinct actin assembly mechanisms in motile cells. J Cell Biol 2006;173:383-394.

17 Yoshioka K, Foletta V, Bernard O, et al. A role for LIM kinase in cancer invasion. Proc Natl Acad Sci USA 2003;100:7247-7252.

18 Rothschild BL, Shim AH, Ammer AG, et al. Cortactin overexpression regulates actin-related protein $2 / 3$ complex activity, motility, and invasion in carcinoma with chromosome 11q13 amplification. Cancer Res 2006;66:8017-8025. 